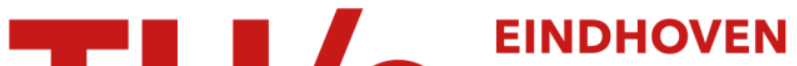 UNIVERSITY OF TECHNOLOGY
}

\section{Electro-optic tuning of a monolithically integrated widely tuneable InP laser with free-running and stabilized operation}

\section{Citation for published version (APA):}

Andreou, S., Williams, K., \& Bente, E. (2020). Electro-optic tuning of a monolithically integrated widely tuneable InP laser with free-running and stabilized operation. Journal of Lightwave Technology, 38(7), 1887-1894. [8894462]. https://doi.org/10.1109/JLT.2019.2952466

DOI:

10.1109/JLT.2019.2952466

Document status and date:

Published: 01/04/2020

\section{Document Version:}

Accepted manuscript including changes made at the peer-review stage

\section{Please check the document version of this publication:}

- A submitted manuscript is the version of the article upon submission and before peer-review. There can be important differences between the submitted version and the official published version of record. People interested in the research are advised to contact the author for the final version of the publication, or visit the $\mathrm{DOI}$ to the publisher's website.

- The final author version and the galley proof are versions of the publication after peer review.

- The final published version features the final layout of the paper including the volume, issue and page numbers.

Link to publication

\section{General rights}

Copyright and moral rights for the publications made accessible in the public portal are retained by the authors and/or other copyright owners and it is a condition of accessing publications that users recognise and abide by the legal requirements associated with these rights.

- Users may download and print one copy of any publication from the public portal for the purpose of private study or research.

- You may not further distribute the material or use it for any profit-making activity or commercial gain

- You may freely distribute the URL identifying the publication in the public portal.

If the publication is distributed under the terms of Article 25fa of the Dutch Copyright Act, indicated by the "Taverne" license above, please follow below link for the End User Agreement:

www.tue.nl/taverne

Take down policy

If you believe that this document breaches copyright please contact us at:

openaccess@tue.nl

providing details and we will investigate your claim. 


\title{
Electro-optic tuning of a monolithically integrated widely tuneable InP laser with free-running and stabilized operation
}

\author{
Stefanos Andreou, Student Member, IEEE, Kevin A. Williams, Member, IEEE, and Erwin A. J. M. \\ Bente, Member, IEEE
}

\begin{abstract}
We report on the free-running and frequency stabilized operation of a ring resonator-tuned full-band tuneable laser. The laser is a monolithically integrated semiconductor ring laser tuneable over $34 \mathrm{~nm}$, from $1522 \mathrm{~nm}$ to $1556 \mathrm{~nm}$, fabricated using a commercially-available, InP-based, active-passive foundry technology. The mode selection is implemented using the Vernier effect by reverse-biasing voltage-controlled electro-optically tuned ring resonators. The laser exhibits a typical intrinsic linewidth of $110 \mathrm{kHz}$ over the $\mathrm{C}$-band and a side-mode suppression ratio (SMSR) exceeding $50 \mathrm{~dB}$. The linewidth over the tuning range does not significantly vary, showing that the tuning mechanism does not cause linewidth broadening. The power dissipated in the tuning elements is $1.4 \mathrm{~mW}$ per ring. Using the same reverse bias voltagecontrolled electro-optic tuning, we also demonstrate the locking of the laser to a high-finesse etalon using Pound-Drever-Hall frequency locking. Our implementation requires a single control loop because of the reverse bias tuning. All deployed tuning effects in the phase modulators have the same sign therefore resulting in a flatter frequency dependent phase response compared to current injection feedback. The control loop has a bandwidth of $500 \mathrm{kHz}$ and a control range of $2.9 \mathrm{GHz}$. We report $1 \mathrm{kHz}$ level linewidth for millisecond observation times for the stabilized laser.
\end{abstract}

Index Terms-laser stability, semiconductor lasers, integrated optics, Indium Phosphide, laser tuning, electro-optic effects.

\section{INTRODUCTION}

$\mathrm{W}$ idely tuneable laser sources with narrow linewidths play a key role in high-speed communications [1], sensing [2], spectroscopy [3], quantum optics [4] and light detection and raging applications [5]. For example, in coherent optical communication systems [6], a 16 quadrature amplitude modulation (QAM) at $40 \mathrm{~Gb} / \mathrm{s}$ a linewidth of $120 \mathrm{kHz}$ is required while upscaling to 64 QAM yields 100 times more stringent requirements on the laser linewidth [7].

Active stabilization schemes have been proposed to dramatically improve the linewidth and frequency noise properties of free-running lasers. This approach is more common in spectroscopy and sensing applications $[3,8]$. The most widely used and powerful technique is the Pound-DreverHall $(\mathrm{PDH})$ frequency locking [9]. In this technique a laser is locked to an external optical cavity by using negative electrical feedback. In the case of diode lasers, two control signals are typically used; one on the semiconductor optical amplifier (SOA) and an external acousto-optic modulator. In [10] an approach which allows the implementation of PDH locking using a single control loop, limited only by the loop delay is demonstrated. This approach deploys a bulk lithium niobate single side-band modulator with bandwidth up to $5 \mathrm{MHz}$.

The most common choice for a stabilized diode laser is an extended cavity diode laser (ECDL); e.g. Littman-Metcalf [11] and Littrow configuration [12] due to its low natural linewidth. A naturally low linewidth greatly relaxes the specifications on the gain and bandwidth of the control loop in order to reach $\sim \mathrm{Hz}$ and sub-Hz level linewidths $[13,14]$. At the same time ECDLs offer another frequency control input to the laser, a piezoelectric transducer controlling the cavity length which is often used for the low bandwidth feedback loop.

Much effort has been put in recent years towards reducing the linewidth of chip-based integrated lasers from several $\mathrm{MHz}$ to the $100 \mathrm{kHz}$ range and below. Different integration strategies have been followed to achieve this goal. In [15-18] for example, monolithically integrated tuneable distributed Bragg reflector (DBR) lasers with $50-100 \mathrm{kHz}$ intrinsic linewidth were demonstrated. The mirrors were sampled and super-mode DBR gratings that were thermally tuned. Tuneable lasers based on current injection tuning have also been reported [19] in the past. However, due to the known linewidth broadening mechanism of the current injection [20] such DBRs are not suited for applications which require very low linewidths. Lasers with even lower intrinsic linewidths have been demonstrated using heterogeneous and hybrid integration of InP with silicon (bonded) and silicon nitrite (butt-coupled). In [21-24], lasers with intrinsic linewidth from tens of $\mathrm{kHz}$ down to sub-kHz have been reported. The reduction of linewidth in these examples is achieved through high quality factor resonators realized with very low loss waveguides. These lasers also deploy thermal tuning since silicon based phase modulators typically deploy mainly carrier effects which are not compatible with low loss waveguides and low linewidth lasers. Phase modulators in $\mathrm{SiN}$ are typically realized by resistive heaters.

The tuning mechanism used in this work differs compared to usual electronic current injection-based or thermo-optic tuning 

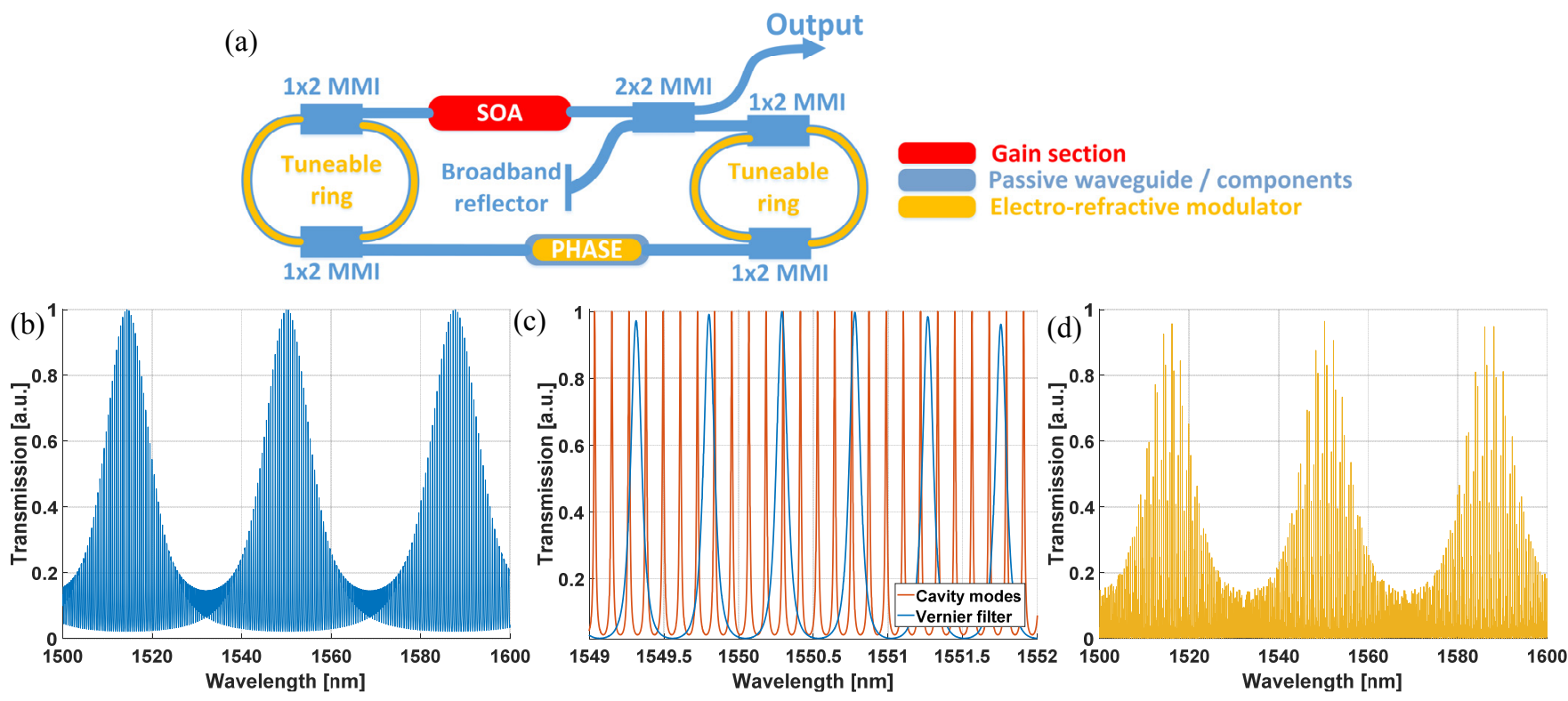

Fig. 1. (a) Schematic of the tuneable ring laser with the two electro-optically tuned ring resonators and phase section and a broadband reflector to enforce unidirectionality, (b) Calculated transmission profile of the Vernier filter (two ring resonators) with total $35 \mathrm{~nm}$ FSR, (c) Close-in of the calculated transmission profile of the Vernier filter (blue) and longitudinal cavity modes (orange), (d) Example of a calculated Vernier filter transmission combined with the cavity mode selection resulting in single mode selection.

as in the work cited in the previous paragraph. As discussed in [25] where the same integration technology [26] is used for a different laser cavity [27], this approach avoids significant onchip heat dissipation. In [25] it is shown that reverse biasing the phase sections results in values for the heat dissipation < $100 \mu \mathrm{W}$ at $8 \mathrm{~V}$. This is significantly lower compared to thermal tuning strategies which typically need several $10 \mathrm{~s}$ of $\mathrm{mW}[5-6$, 23]. The origin of the heat dissipation is the reverse bias current due to the carrier depletion in the intrinsic region which increases at higher voltages. This tuning mechanism can also enable faster tuning compared to thermal tuning due to the weaker slow transient thermal effects which are involved. Furthermore, contrary to current injection tuning no significant propagation losses due to free-carrier absorption takes place. As shown in [28], low additional propagation losses occur only at high voltages due to electro-absorption. In fact, at low voltages $(<5 \mathrm{~V})$ losses are even slightly reduced due to free-carriers depletion.

In this work, we demonstrate an InP monolithically integrated unidirectional single mode ring laser fabricated using an openaccess InP foundry technology. The laser deploys the Vernier effect from two periodical spectral filters for its single-mode operation and it is tuned using voltage controlled electro-optic effects. We present tuning and intrinsic linewidth results. The average dissipated power in the tuning mechanism is quantified. We also demonstrate the stabilization of the same laser using the PDH frequency locking.

\section{UNIDIRECTIONAL VERNIER RING LASER USING INP ACTIVE- PASSIVE INTEGRATION}

A schematic of the laser is presented in Fig. 1(a). The ring cavity includes a $1 \mathrm{~mm}$ long SOA with InGaAsP multiquantum-well based material. The SOA is based on a shallow etched ridge waveguide. The two ring resonators have a radius of $\sim 200 \mu \mathrm{m}$ and the power coupling is implemented using $2 \mathrm{x} 1$ multimode interference (MMI) couplers with 50\% splitting ratio. The typical minimum bending radius in this technology is about $100 \mu \mathrm{m}$ with losses $0.1 \mathrm{~dB} / 90^{\circ}$. Here however we do not opt for the minimum possible radius in order to obtain sufficient tuning length and not to limit the tuning range of the laser. The coupling ratio is not optimal but these MMIs are readily available in this foundry technology. The total circumference of a single ring resonator, including the length of the MMIs, is $1.4 \mathrm{~mm}$ and $1.419 \mathrm{~mm}$. A $0.4 \mathrm{~mm}$ long electro-optic phase modulator section is also included in the laser cavity to facilitate independent tuning the cavity modes.

The two ring resonators and the phase section can be tuned by applying a reverse bias voltage. They are both deeply etched ridge waveguides, their cross-section is a vertical PIN diode and act as electro-refractive modulators (ERM). The nonintentionally-doped guiding layer is bulk InGaAsP quaternary material with bandgap at $1.25 \mu \mathrm{m}$. The effective index changes are a result of both field (Pockels and Kerr) and carrier (plasma/carrier depletion and band-filling) effects. The addition of these effects results in a modulator efficiency of about $15^{\circ} / \mathrm{Vmm}$ (specified by the foundry [26]) for TE polarized light.

The power out-coupling is implemented by a 2x2 MMI with 85-15 splitting ratio. The out-coupling power percentage is $15 \%$. The unidirectional operation of the ring laser is ensured by an extra-cavity broadband reflector (Fig. 1(a)) which couples the amplified spontaneous emission (ASE) in the anticlock-wise propagating mode to the clock-wise mode. The broadband reflector is a multi-mode interference reflector. The same approach has been followed in the tuneable laser reported in [27]. The total physical length of the cavity is $5.9 \mathrm{~mm}$ calculated from the laser mask layout corresponding to a cavity mode free-spectral range (FSR) of $13.5 \mathrm{GHz}(0.108 \mathrm{~nm})$.

We use the Vernier effect [29] of the two ring resonators with slightly different circumference to increase the FSR of the filter and to select a single lasing mode within the modal gain 
bandwidth. The enhanced FSR from the Vernier effect is calculated by

$$
\Delta \lambda_{F S R, \text { Vernier }}=\frac{\Delta \lambda_{F S R 1} \Delta \lambda_{F S R 2}}{\Delta \lambda_{F S R 1}-\Delta \lambda_{F S R 2}}
$$

where $\Delta \lambda_{F S R 1}$ and $\Delta \lambda_{F S R 2}$ are the FSR of the individual ring resonators. The FSR of an individual ring resonator is given by $\Delta \lambda_{F S R}=\lambda^{2} / n_{g} L$, where $n_{g}$ is the group index, $L$ the circumference of a single ring and $\lambda$ the wavelength. Ideally the detuning of the two rings should be large enough such that the tuning range is only limited by the modal gain bandwidth of the SOA and not the FSR of the Vernier intra-cavity filter. Practically however, if the full-width at half-maximum (FWHM) of the resonances of the rings is not narrow enough, a smaller detuning of the rings could lead to degraded SMSR because the neighbouring cavity modes are not adequately suppressed. The radius difference between the two rings was chosen to be $3 \mu \mathrm{m}$, resulting in a path length difference of $19 \mu \mathrm{m}$. The radii difference yields a Vernier FSR of about $35 \mathrm{~nm}$ as can be seen from the transmission spectra of the two rings in Fig. 1(b). This FSR ensures that the laser tuning range is larger than $30 \mathrm{~nm}$ therefore covering the span of a single band and does not compromise single-mode operation.

The transmission spectrum for the two ring filters is calculated and shown in Fig. 1(c) (blue) for a narrow wavelength span where also the longitudinal cavity modes (orange) are presented. The spacing of the cavity modes and the ring filter modes is such that a cavity mode falls within a ring filter transmission peak every $4 \mathrm{x} \Delta \lambda_{F S R}$. Effectively the cavity modes within this $4 \mathrm{x} \Delta \lambda_{F S R}$ range are suppressed by the ring filters. The gain difference between the lasing mode and the neighboring cavity mode falling within a ring resonator transmission peak (which is $4 \times \Delta \lambda_{F S R}$ away) is about $8 \%$. This transmission difference is adequate to ensure single mode operation of the laser. The total cavity transmission response, including of the two ring resonators and the laser cavity modes, is shown in Fig. 1(d).

\section{LASER SIMULATION}

Using the commercial simulation software PicWave (Photon Design), we have performed full circuit-level time-domain simulations. The simulations are based on the time-domain travelling-wave method in which only the slowly-varying optical field envelope is propagated in the spatially discretized laser cavity. The simulations were performed for a time span of $100 \mathrm{~ns}$ over a wavelength range of $70 \mathrm{~nm}$. The spectra correspond to the laser steady-state and any transients have been discarded.

An example of the normalized output spectrum is shown in Fig. 2(a). Single mode behavior with a SMSR of slightly below $60 \mathrm{~dB}$ is predicted by the simulations. The cavity mode structure resulting from the two rings and the cavity modes is also visible. The bias conditions of the two rings were varied and the normalized simulated output spectra are shown in Fig. 2(b). The model predicts a tuning range of $32 \mathrm{~nm}$ with SMSR over $45 \mathrm{~dB}$ across the tuning range.
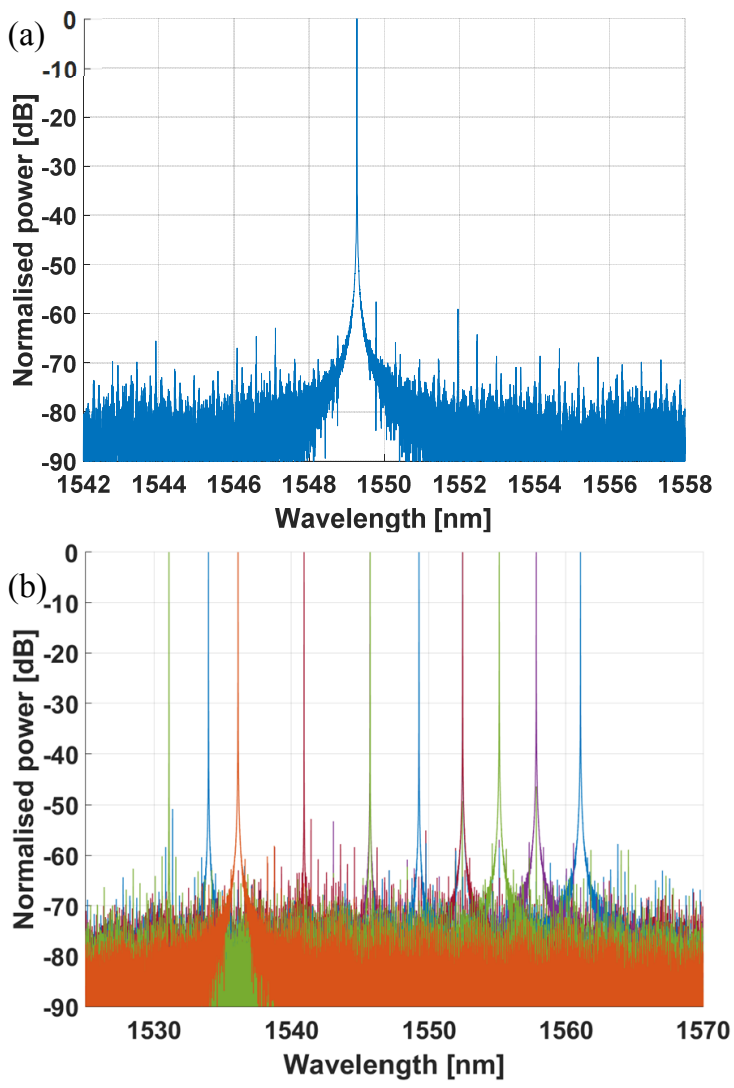

Fig. 2. (a) Single simulated spectrum at $120 \mathrm{~mA}$ SOA current with single mode operation at $1549.5 \mathrm{~nm}$, (b) Multiple overlapping simulated output spectra of the tuned laser around the C-band at $120 \mathrm{~mA}$ SOA current. The tuning range is predicted to be around $32 \mathrm{~nm}$.

\section{LASER CHARACTERIZATION - SPECTRAL PERFORMANCE AND ELECTRO-OPTIC TUNING}

The laser was fabricated using a commercially available active-passive InP-based integration technology [30] in the framework of a multi-project wafer run by Smart Photonics [26]. A microscopic image of the laser is shown in Fig. 3(a). Its footprint is $2.17 \times 0.56 \mathrm{~mm}^{2}$. The laser was characterized at $18^{\circ} \mathrm{C}$ using a water-cooler based temperature stabilized mount. The waveguide from the laser output is angled with respect to the chip facet to suppress back-reflections to the laser cavity. The chip facet was also coated with anti-reflection coating to further suppress back-reflections. The laser output light was coupled out of the chip using a single-mode lensed fibre as shown in Fig. 3(a). Typical coupling losses between the chip facet and the lensed fibre due to the mode mismatch are $\sim 4 \mathrm{~dB}$.

The SOA current is provided by a laser diode controller (Thorlabs LDC8010) and the output optical power is monitored by a power sensor (Agilent $81635 \mathrm{~A}$ ). The two ring resonators are reversed biased from $0-10 \mathrm{~V}$ with $0.5 \mathrm{~V}$ step using a source-meter (Keithley 2602B). The photo-current flowing through the two ring resonators is recorded for the different biasing conditions while the laser tuning is performed. The phase section was not used for tuning and it was left unbiased. The phase section could be used as a solution for the laser mode-hopping. However, this is not investigated here. The 
(a)

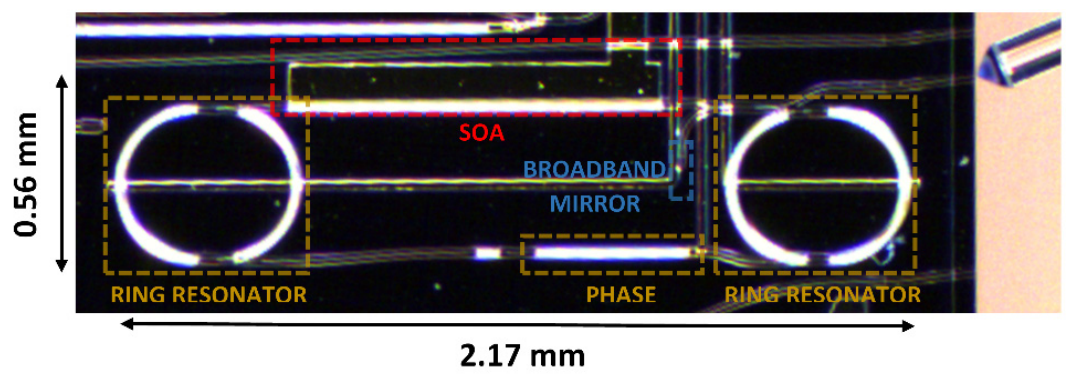

(b)

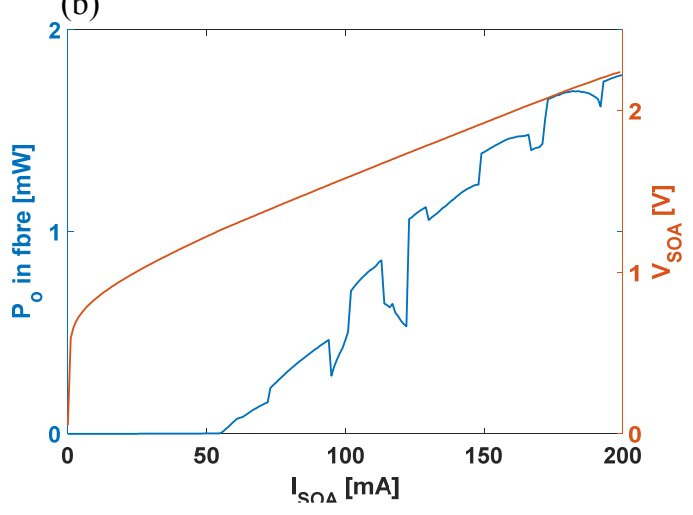

Fig. 3. (a) Microscope image of the fabricated laser with footprint of $\sim 2.17$ x $0.56 \mathrm{~mm}^{2}$, (b) Optical output power in fibre (blue) and voltage (orange) of the SOA as a function of SOA injected current at $18{ }^{\circ} \mathrm{C}$. The kinks on the light-current curve are due to laser mode-hopping.

optical spectra are recorded with two optical spectrum analyzers (OSA); the first one with a resolution bandwidth of $0.05 \mathrm{~nm}$ (ANDO AQ6315A) and the second with a highresolution spectrum analyzer (APEX 2641B) $5 \mathrm{MHz}$ resolution bandwidth. Two OSAs are used because the shortest wavelength of the high-resolution OSA wavelength range is limited to $1528 \mathrm{~nm}$ but it is more suitable for a more detailed image of the output spectra.

In Fig. 3(b) the optical output power in fibre as a function of the gain element current in the cavity is presented. The kinks in the light-current (LI) curve are due to mode-hopping of the laser as it is thermally tuned while the SOA current is being swept. The threshold current is about $56 \mathrm{~mA}$, corresponding to a current density of $2.8 \mathrm{kA} / \mathrm{cm}^{2}$. The optical power in fibre reaches about $1.8 \mathrm{~mW}$ at $200 \mathrm{~mA} \mathrm{SOA}$ current and at a current density of $10 \mathrm{kA} / \mathrm{cm}^{2}$. The series resistance derived from the voltage-current curve is about $7 \mathrm{Ohms}$.

In Fig. 4(a) tuning spectra of the laser operated at $120 \mathrm{~mA}$ SOA current with resolution bandwidth $0.05 \mathrm{~nm}$ are presented. The tuning range reaches $34 \mathrm{~nm}$, from $1522 \mathrm{~nm}$ to $1556 \mathrm{~nm}$. The peak power fluctuations over the tuning range result from imperfect tuning of the laser since the tuning steps are rather coarse $(0.5 \mathrm{~V})$. During the tuning of the laser we noticed some difficulty in tuning with accuracy of $\sim 100 \mathrm{MHz}(\sim 1 \mathrm{pm})$. This may be attributed to spurious reflections due to the MMIs, however more investigation is necessary to draw conclusions. In case deemed necessary low reflection MMIs could be used instead. In Fig. 4(b) a more detailed image of the output optical spectrum at $1548.6 \mathrm{~nm}$ is presented with $5 \mathrm{MHz}$ resolution bandwidth. The closest neighboring mode to the lasing mode is three times the ring FSR away which is just visible above the noise floor of the instrument. This is in line with the design considerations discussed in section II. The SMSR is about $59 \mathrm{~dB}$ in this case. The SMSR for spectra over the tuning range recorded with $5 \mathrm{MHz}$ is presented in Fig. 5. The SMSR of the spectra exceeds $50 \mathrm{~dB}$ across the whole tuning range.

In Fig. 6 we present the dissipated tuning power (blue) and reverse bias current (orange) of the two ring resonators as a function of the rings' reverse bias voltage. The dissipated power levels vary over 4 orders of magnitude over the $10 \mathrm{~V}$ tuning range; from some $\mu \mathrm{W}$ at low voltages to a maximum of $\sim 14$ $\mathrm{mW}$ at $10 \mathrm{~V}$ bias. This is the maximum voltage applied for obtaining the full tuning range. The average tuning power consumption for a single ring over the $10 \mathrm{~V}$ range is $1.4 \mathrm{~mW}$.
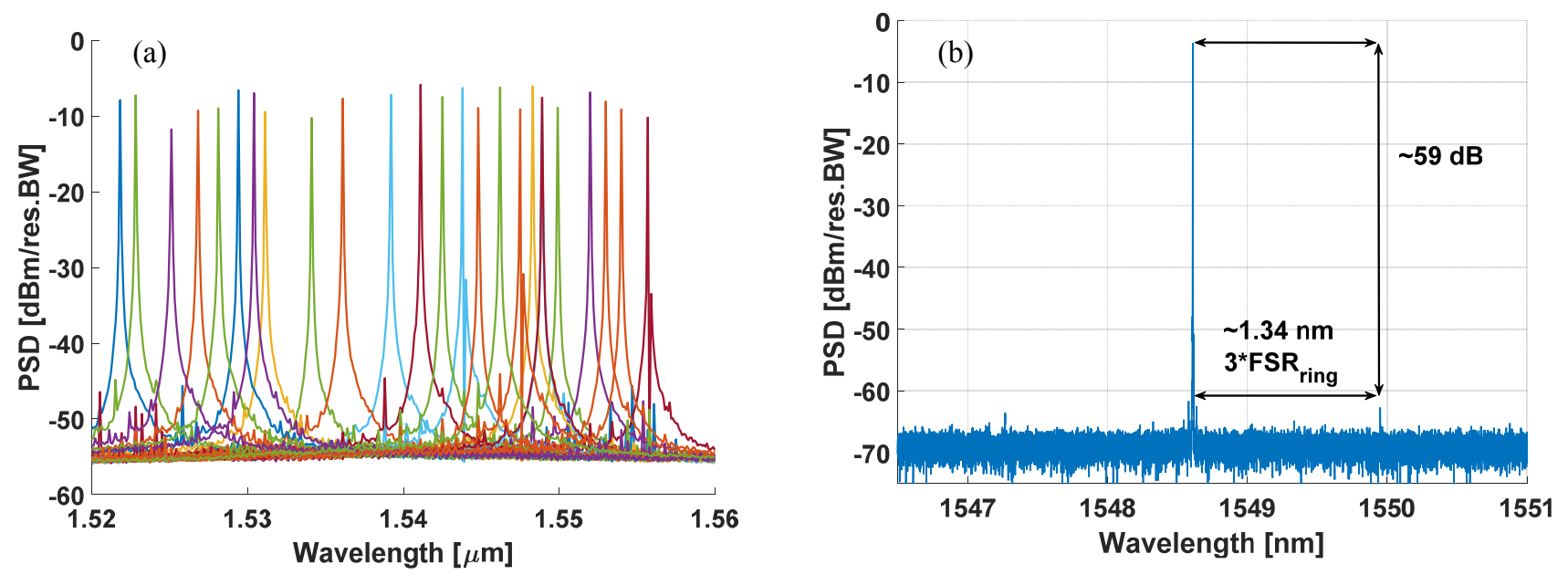

Fig. 4. (a) Optical spectra showing a tuning range of $34 \mathrm{~nm}$ (res. BW: $0.1 \mathrm{~nm}$ ) with $120 \mathrm{~mA}$ SOA current, (b) Single spectrum at $1548.7 \mathrm{~nm}$ (recorded with a high resolution optical spectrum analyzer (res.bw: $5 \mathrm{MHz}$ ) with $59 \mathrm{~dB}$ SMSR and the closest side-mode $\sim 1.34 \mathrm{~nm}$ apart, corresponding to three times the ring FSR. 


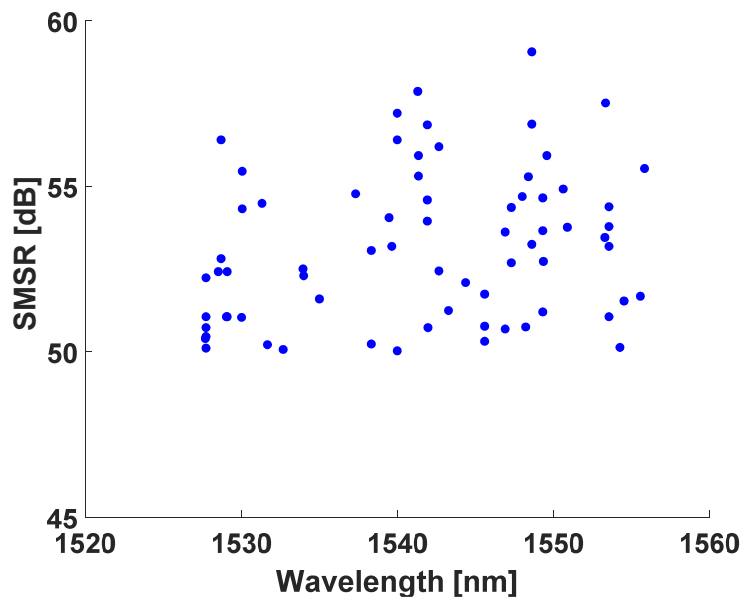

Fig. 5. The SMSR of the laser as at different lasing wavelengths (the spectra were recorded with $5 \mathrm{MHz}$ res. bw).

The maximum photo-current occurring for $-10 \mathrm{~V}$ is $1.4 \mathrm{~mA}$.

\section{INTRINSIC LINEWIDTH}

The intrinsic linewidth of the laser was determined by measuring its frequency noise power spectral density [31]. For these measurements, the SOA current was provided by a lownoise battery power current source (ILX LDX-3620B). The measurements were performed with a frequency noise measurement setup from OEwaves (OE4000) capable of measuring frequency noise corresponding to linewidth of $10 \mathrm{~Hz}$. The noise floor of the instrument is therefore much lower that the measured frequency noise. Before entering the frequency noise measurement setup the optical signal was amplified using a low-noise figure $(4 \mathrm{~dB}$, defined by the manufacturer) erbium-doped fibre amplifier (Keopsys CEFAC-HG-SM-50-B130) to obtain sufficient optical power ( $>5 \mathrm{dBm}$ for the frequency noise measurements). The EDFA ASE was filtered using a narrow passband filter with $0.4 \mathrm{~nm}$

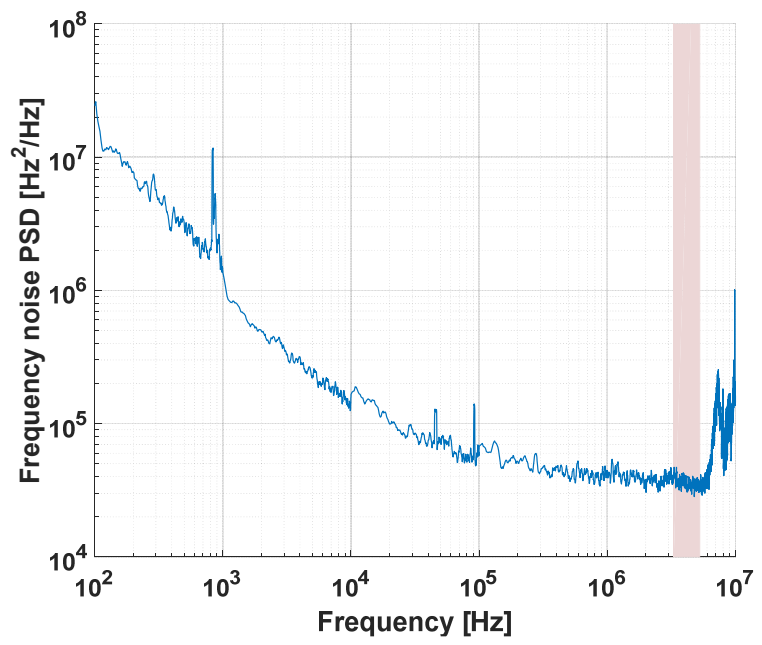

Fig. 7. Power spectral density of the frequency noise of the laser at $120 \mathrm{~mA}$ SOA current lasing at $1548.6 \mathrm{~nm}$. The orange area indicates the white part of the frequency noise which multiplied by $\pi$ yields an intrinsic linewidth of $109 \mathrm{kHz}$.

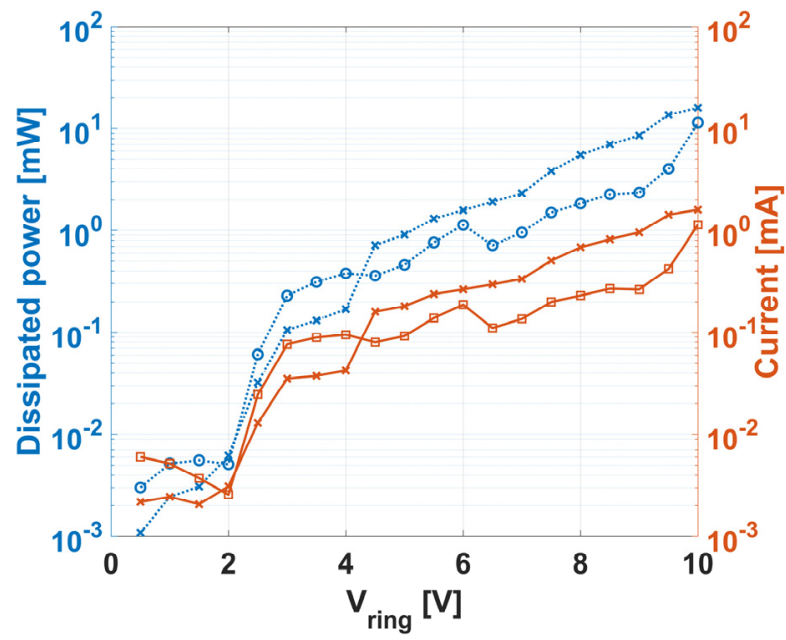

Fig. 6. The tuning power (blue) and reverse bias current (orange) for the two ring resonators starts from $1 \mu \mathrm{W}$ at low voltages and increases to a maximum of $\sim 14 \mathrm{~mW}$ for $10 \mathrm{~V}$ reverse bias voltage.

width.

The single-sided frequency noise of the laser at $1548.6 \mathrm{~nm}$ and at $120 \mathrm{~mA}$ SOA current is presented in Fig. 7. The laser noise exhibits a typical $1 / f$ behavior due to technical noise. The $1 / f$ noise slope gradually decreases in the range from $100 \mathrm{~Hz}$ to $\sim 300 \mathrm{kHz}$. The flat-band (white) frequency noise above $100 \mathrm{kHz}$ is attributed to the ASE of the laser determining the laser Lorentzian linewidth. We average the noise level in the range indicated by the shaded area in Fig. 7. The frequency noise power spectral density level is $34.7 \mathrm{~Hz}^{2} / \mathrm{Hz}$. This value can then be multiplied by $\pi$ to calculate the laser Lorentzian (intrinsic) linewidth. The Lorentzian linewidth is $109 \mathrm{kHz}$ for this wavelength, $1548.6 \mathrm{~nm}$. The spike near $10 \mathrm{MHz}$ is due to the laser diode controller.

The intrinsic linewidths calculated from the frequency noise measurements at the same SOA current $(120 \mathrm{~mA})$ at several lasing wavelengths are presented in Fig. 8. The Lorentzian linewidth remains below $110 \mathrm{kHz}$ for all wavelengths up to $1550 \mathrm{~nm}$ with a minimum of $95 \mathrm{kHz}$. This slightly lower linewidth value could be due to lower $\alpha$-factor caused by the

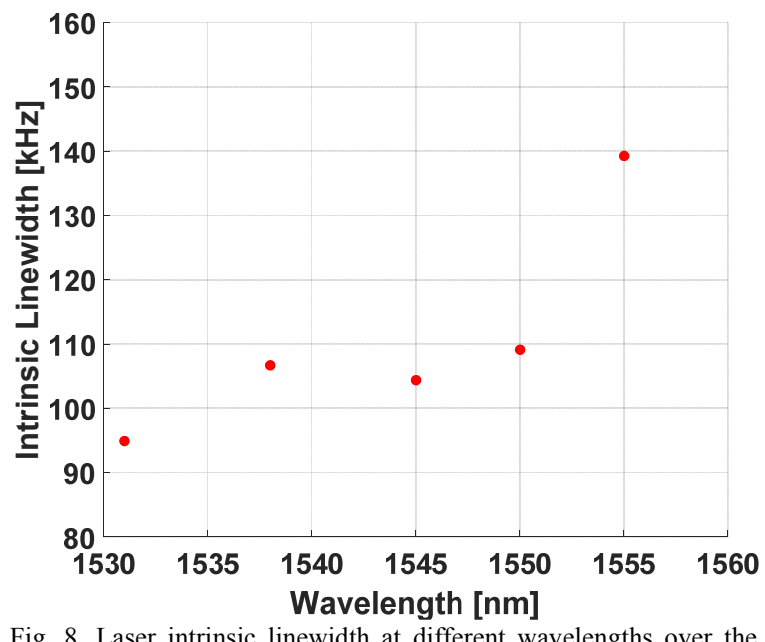

Fig. 8. Laser intrinsic linewidth at different wavelengths over the tuning range. 
negative wavelength detuning [32, 33]. Only at $1555 \mathrm{~nm}$, at the longer wavelength edge of the tuning range the linewidth increases to about $140 \mathrm{kHz}$. Near the edge of the tuning range the SOA operates at higher carrier density due to lower modal gain. This operating condition translates to higher ASE level and therefore higher intrinsic linewidth.

\section{DISCUSSION ON LASER CHARACTERIZATION}

As discussed in Section II, voltage-controlled electro-optic tuning is not expected to introduce significant thermal effects and subsequent tuning. From Fig. 6, we observe that the dissipated tuning power is indeed significantly lower compared to typical thermal tuning, at least for the largest part of the voltage range from $0-10 \mathrm{~V}$. More specifically, the power dissipation rises above $1 \mathrm{~mW}$, only for bias voltages $>5 \mathrm{~V}$ and it reaches a maximum of $10-15 \mathrm{~mW}$ only at the above $8-9 \mathrm{~V}$. It is only at this maximum level, that the power starts becoming comparable with thermal tuning power. A decrease in the dissipated power would require a decrease in the applied reverse bias voltage. Such restriction would however compromise the laser tuning range in the current design. Alternatively the circumference of the tuneable ring filters could be chosen longer such the same phase shift with the current design could be obtained with lower voltages. This modification is in fact favourable due to the linear phase shift with respect to voltage and hyper-linear decrease of photocurrent with decreasing voltage. Nevertheless in this case, the average tuning power $(1.4 \mathrm{~mW})$ is at least an order of magnitude lower than typical thermal tuning power.

Furthermore, electronic tuning is well-known for introducing intra-cavity losses and significant carrier concentration changes in the cavity leading to increased phase noise. All these effects can increase the laser linewidth by almost an order magnitude. In Fig. 8 we show that the intrinsic linewidth of our tuneable laser under reverse bias voltage conditions ranging from 0 to $10 \mathrm{~V}$ does not significantly change. We can therefore conclude that our tuning mechanism has a negligible impact on the laser intrinsic linewidth. We attribute this behaviour to the following:

1) The carrier concentration fluctuation caused by the shot noise due to the voltage-controlled tuning is much lower compared to current-injection tuning. This is a result of the low impendence of voltage sources compared to the high impendence of current sources. Ultimately, lower carrier concentration fluctuations also mean smaller variations in the effective index of the guiding mode and therefore lower frequency noise.

2) In a reverse bias configuration the carrier lifetime in the mode volume reduces from several hundred picoseconds to a couple of tens of picoseconds. This reduction in carrier lifetime leads to a lower carrier concentration. Since the generation rate of these carriers remains the same, the carrier concentration fluctuations also reduce.

3) The Schawlow-Townes-Henry theory on laser linewidth states that the linewidth of a laser due to ASE is inversely proportional to the intra-cavity losses. Reverse biasing does not significantly alter the passive loss of the ERMs (and by extension the intra-cavity losses) therefore the intrinsic linewidth of the laser does not degrade either.

\section{Stabilization OF THE INTEGRATED TUNEABLE LASER}

In this section we apply PDH stabilization to the integrated extended cavity tuneable diode laser.

\section{A. Pound-Drever-Hall frequency stabilization setup}

In Fig. 9 a schematic of the PDH frequency stabilization setup is presented. The laser is operated at $18^{\circ} \mathrm{C}$ controlled by a watercooler with $120 \mathrm{~mA}$ SOA current supplied by a lownoise battery powered current source (ILX LDX-3620B). It is tuned at $1548.6 \mathrm{~nm}$ in single mode operation. The external optical cavity is a highly stable $\left(47 \mathrm{kHz} /{ }^{\circ} \mathrm{C}\right) \mathrm{Fabry}-\mathrm{Perot}$ cavity with FWHM of $\sim 700 \mathrm{kHz}$ and FSR of $\sim 3.125 \mathrm{GHz}$ manufactured by StableLasers. The laser output is phase modulated at $60 \mathrm{MHz}$ by a lithium niobate phase modulator. The sidebands reflected from the cavity are detected by a photodetector (Femto OE-300-IN-01) with bandwidth and transimpedance gain of $175 \mathrm{MHz}$ and $10^{3} \mathrm{~V} / \mathrm{A}$ respectively. Its output is down-converted to baseband and fed to a proportionalintegral-derivative (PID) controller.

The PID controller, the local oscillator, the mixer and phase shifter are all commercial electronics by Toptica (PDD110, FALC110). The PID controller includes two outputs, low $\left(f_{b w}<10 \mathrm{~Hz}\right)$ and high $\left(10 \mathrm{~Hz}<f_{b w}<10 \mathrm{MHz}\right)$ bandwidth. The cut-off frequencies used for the two integrators and the differentiator in the fast branch are $6.5 \mathrm{kHz}, 170 \mathrm{kHz}$ and $190 \mathrm{kHz}$ respectively. The two outputs of the PID controller are summed with a DC voltage using a summing amplifier (SRS SIM980) with a bandwidth of $1 \mathrm{MHz}$. The summing amplifier output biases a battery-powered opto-coupler which is used for electrical isolation of the laser. Based on the capacitance of the large-area photodetector of the opto-coupler and the series resistance connected to it, we calculate a bandwidth of about $500 \mathrm{kHz}$. The opto-coupler output is biasing with a negative voltage the $400 \mu \mathrm{m}$ long phase section of the tuneable laser which independently controls the cavity modes.

The laser output is split by a $90 / 10$ fibre splitter. The $90 \%$ of the power is used for the PDH locking system. The remaining $10 \%$ of the laser output power is used for characterization of the laser output using the delayed self-heterodyne method with a $25 \mathrm{~km}$ delay line and the frequency noise power spectral density. Before the two characterization setups an EDFA with a narrow bandpass filter were used to amplify the optical signal and obtain sufficient optical power. The details for the EDFA and filter were the same as the ones described in section $\mathrm{V}$.

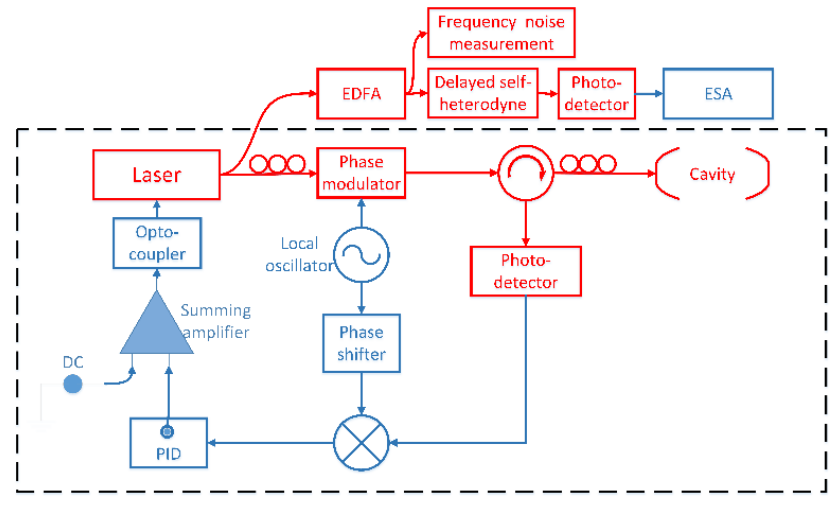

Fig. 9. Schematic of the PDH frequency stabilization setup used for the locking of the integrated tunable laser. 


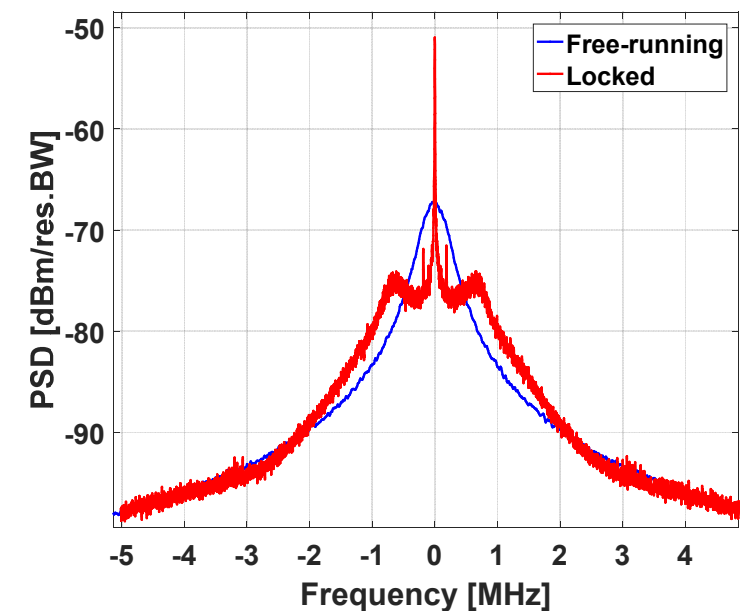

Fig. 10. Electrical spectra (average of 20 measurements) of the freerunning (blue) and locked (red) laser from the delayed-self-heterodyne setup with $25 \mathrm{~km}$ delay, recorded with $3 \mathrm{kHz}$ resolution bandwidth and $300 \mathrm{~Hz}$ video bandwidth.

\section{B. Frequency stabilization results and discussion}

From the open-loop error signal we have estimated the sensitivity of the control system and laser which is about $0.29 \mathrm{GHz} / \mathrm{V}$. This is slightly higher but nevertheless consistent with the expected sensitivity $(0.225 \mathrm{GHz} / \mathrm{V})$ for the $400 \mu \mathrm{m}$ with the assumed efficiency of $15^{\circ} / \mathrm{Vmm}$. The typical voltage range of the reverse bias voltage is $10 \mathrm{~V}$ which yields a control range of about $2.9 \mathrm{GHz}$.

In Fig. 10 the electrical spectra from the delayed selfheterodyne setup are presented for both the free-running (blue) and locked laser (red). The spectra are an average of twenty measurements which were recorded with a resolution bandwidth of $3 \mathrm{kHz}$ and video bandwidth of $300 \mathrm{~Hz}$. The freerunning laser exhibits a linewidth of about $230 \mathrm{kHz}$ which has a Gaussian lineshape. This lineshape indicates that the measured linewidth is dominated by technical noise $(1 / f)$. This linewidth is considerably wider than the intrinsic linewidth reported in section $\mathrm{V}$. The locked laser has a more complex lineshape (Fig. 10, red trace). It shows a substructure due to the frequency noise reduction within the feedback loop bandwidth. The width of the mode is much narrower compared to the freerunning operation therefore the power spectral density is also much higher compared to the free-running laser.

The effect of the locking system on the laser is better quantified from the laser frequency noise power spectral density. In Fig. 11 the power spectral density of the frequency noise of the laser is plotted. The frequency noise of the freerunning laser exhibits the same behaviour discussed in section $\mathrm{V}, 1 / f$ noise in low frequencies and white noise at higher. The locked laser frequency noise is significantly reduced in the range from $100 \mathrm{~Hz}$ up to $500 \mathrm{kHz}$. At the higher frequency, the two frequency noise traces cross each other because the gain of the control loop is unity. The bandwidth limitation originates from the $\mathrm{RC}$ constant of the photodetector in the opto-coupler which is expected to have a similar bandwidth. The wide peak around $800 \mathrm{kHz}$ is due to the phase lag of the control loop at those frequencies. Between $1-10 \mathrm{kHz}$ we observe the highest noise suppression reaching $30 \mathrm{~dB}$. The noise suppression reduces for higher frequencies. Below $1 \mathrm{kHz}$

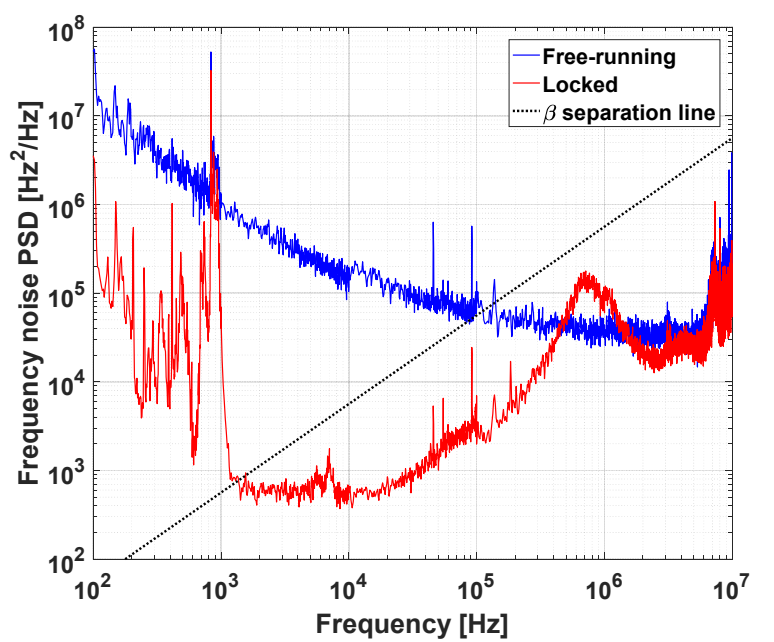

Fig. 11. Power spectral density of the frequency noise of the free-running (blue) and locked (red) laser.

we observe spurious peaks which are not effectively supressed by the feedback loop. The most prominent peak is at $900 \mathrm{~Hz}$. The origin of these noise peaks is unknown.

In Fig. 11, we also plot the $\beta$-separation line [34] which is defined as

$$
\beta_{\text {sep }}(f)=8 \frac{\ln (2) f}{\pi^{2}}
$$

and it indicates the portion of the frequency noise which contributes to the laser linewidth. This part is the one for which $S_{v}(f)>\beta_{\text {sep }}(f)$ holds, where $S_{v}(f)$ is the frequency noise of the laser and $\beta_{\text {sep }}(f)$ the $\beta$-separation line. Its crossing with the frequency noise of the free-running laser is at about $100 \mathrm{kHz}$. We can therefore conclude that our loop has sufficient bandwidth to suppress the frequency noise contributing to the laser linewidth and effectively reduce linewidth. The width of the lineshape can be calculated from the measured frequency noise by the simple relation [34]

$$
\Delta v=\sqrt{8 \ln (2) A}
$$

where $A$ is the surface for which $S_{v}(f)>\beta_{\text {sep }}(f)$ holds. The calculated linewidth is depended on the integration interval or in other words the observation time.

We numerically integrate the measured frequency noise and calculate $A$ for the locked laser frequency noise. We find that the expected linewidth is below $\mathrm{kHz}$ for observation times shorter than $1 \mathrm{~ms}$. This is in fact consistent with our observations from the electrical spectra of the delayed selfheterodyne method recorded with $3 \mathrm{kHz}$ resolution bandwidth where the width of the lineshape is resolution limited. The calculated linewidth however steeply increases from $1 \mathrm{kHz}$ to about $40 \mathrm{kHz}$ for observation times between $1 \mathrm{~ms}$ and $1.25 \mathrm{~ms}$ due to the prominent spurious peaks at $900 \mathrm{~Hz}$. Finally comparing the calculated linewidth of the free-running and locked lasers for $1 \mathrm{~ms}$ observation time yields an improvement of more than 200 since the free-running linewidth is about $230 \mathrm{kHz}$ (Fig. 10, blue trace). 


\section{Conclusions}

We demonstrate a tuneable ring laser deploying the Vernier effect with two intra-cavity ring resonators fabricated using a commercially available open-access InP integration technology. This is the first ring resonator tuned full-band tuneable laser in such a platform to the best of our knowledge. The laser exhibits a tuning range of $34 \mathrm{~nm}$ with a typical linewidth of $110 \mathrm{kHz}$ and an SMSR over $50 \mathrm{~dB}$ over the whole range. The electro-optic tuning does not cause broadening of the intrinsic linewidth and the average power dissipation in the tuning element over the $10 \mathrm{~V}$ range is $1.4 \mathrm{~mW}$ per ring. We demonstrate the stabilization of the same laser using the PDH locking technique down to $1 \mathrm{kHz}$ linewidth for millisecond observation times. Based on the laser electro-optic tuning, the frequency locking is implemented with a single voltagecontrolled feedback loop and a bandwidth of $500 \mathrm{kHz}$. The negative electrical feedback is applied on the reverse biased intra-cavity phase section.

\section{ACKNOWLEDGMENT}

We acknowledge the Netherlands organization for scientific research (NWO) that funded this work under the grant 13930 and the United States Air Force Office of Scientific Research (AFOSR) for the grant FA9550-18-1-0015.

\section{REFERENCES}

[1] C. R. S. Fludger, E. S. Vercelli, G. Marenco, A. Della Torre, T. Duthel, and T. Kupfer, "1Tb/s Real-Time $4 \times 40$ Gbaud DP-16QAM SuperChannel Using CFP2-ACO Pluggable Modules Over $625 \mathrm{~km}$ of Standard Fiber," J. Lightwave Technol., vol. 35, no. 4, 949-954, 2017.

[2] L. He, S. K. Ozdemir, J. Zhu, W. Kim, and L. Yang, "Detecting single viruses and nanoparticles using whispering gallery microlasers," Nat. Nanotechnol., vol. 6, no. 7, pp. 428-432, 2011.

[3] A. Hemmerich, D. McIntyre, D. Schropp, D. Meschede, and T. Hansch, "Optically stabilized narrow linewidth semiconductor laser for high resolution spectroscopy," Opt. Commun., vol. 75, no. 2, pp. 118 - 122, 1990.

[4] J. Benhelm, G. Kirchmair, C. F. Roos, and R. Blatt, "Towards faulttolerant quantum computing with trapped ions," Nat. Phys., vol. 4, pp. 463-466, 2008

[5] E. Dale, W. Liang, D. Eliyahu, A. Savchenkov, V. Ilchenko, A. B. Matsko, D. Seidel, and L. Maleki, "On phase noise of self-injection locked semiconductor lasers", Imaging and Applied Optics, 2014, paper JTu2C. 3

[6] M. Lauermann et al., "Multi-Channel, Widely-Tunable Coherent Transmitter and Receiver PICs Operating at 88Gbaud/16-QAM," Proc. OFC, Los Angeles, 2017.

[7] M. Seimetz, "Laser linewidth limitations for optical systems with highorder modulation employing feed forward digital carrier phase estimation," OFC/NFOEC 2008 - 2008 Conf. Opt. Fiber Commun. Fiber Opt. Eng. Conf., pp. 2-4, 2008.

[8] J. Chen, Q. Liu, X. Fan and Z. He, "Ultrahigh resolution optical fiber strain sensor using dual Pound - Drever- Hall feedback loops," Optics Letters, vol. 41, no. 5, pp. 1066-1069, 2016.

[9] R. W. P. Drever, J. L. Hall, F. V. Kowalski, J. Hough, G. M. Ford, A. J. Munley and H. Ward, "Laser phase and frequency stabilization using an optical resonator,” Applied Physics B, vol. 31, no 12, pp. 97-105, Jun. 1983.

[10] D. Gatti et al., "Wide-bandwidth Pound - Drever - Hall locking through a single-sideband modulator," Opt. Lett., vol. 40, no. 22, pp. 5176-5179, 2015.

[11] Michael G. Littman and Harold J. Metcalf, "Spectrally narrow pulsed dye laser without beam expander," Appl. Opt., 17, 2224-2227, 1978.

[12] C. E. Wieman and L. Hollberg, "Using diode lasers for atomic physics," Rev. Sci. Instrum., vol. 162, no. 1, 1-20, 1991.
[13] A. D. Ludlow, X. Huang, M. Notcutt, T. Zanon-Willette, S. M. Foreman, M. M. Boyd, S. Blatt, and J. Ye, "Compact, thermal-noise-limited optical cavity for diode laser stabilization at 1×10-15," Opt. Lett. 32, 641-643, 2007.

[14] H. Stoehr, F. Mensing, J. Helmcke, and U. Sterr, "Diode laser with $1 \mathrm{~Hz}$ linewidth," Opt. Lett., 31, 736-738, 2006.

[15] M. C. Larson et al., "Narrow linewidth sampled-grating distributed Bragg reflector laser with enhanced side-mode suppression," $38^{\text {th }}$ Optical Fibre Communications Conference, M2D.1, 2015.

[16] S.C. Davies, et al., "Narrow Linewidth, High Power, High Operating Temperature Digital Supermode Distributed Bragg Reflector Laser", 39th European Conference on Optical Communication, Th.1.B.3, 2013.

[17] Y. Matsui et al., "Narrow linewidth tunable semiconductor laser", Compound Semiconductor Week, MoC4-1, 2016.

[18] M. C. Larson, "Narrow linewidth tunable DBR lasers", IEEE International Semiconductor Laser Conference, TuC2, 2016.

[19] A. J. Ward, D. J. Robbins, G. Busico, E. Barton, L. Ponnampalam, J. P. Duck, N. D. Whitbread, P. J. Williams, D. C. J. Reid, A. C. Carter, and M. J. Wale, "Widely tunable DS-DBR laser with monolithically integrated SOA: design and performance," IEEE J. Sel. Topics Quantum Electron., vol. 11, no. 1, pp. 149-156, Jan./Feb. 2005.

[20] J. Buus, M.C. Amann, D. J. Blumenthal, Tunable laser diodes and related optical sources, $2^{\text {nd }}$ Edition, Wiley, 2005.

[21] Y. Fan, R. M. Oldenbeuving, C. G. Roeloffzen, M. Hoekman, D. Geskus, R. G. Heideman, and K. Boller, "290 Hz Intrinsic Linewidth from an Integrated Optical Chip-based Widely Tunable InP-Si3N4 Hybrid Laser," Conference on Lasers and Electro-Optics, OSA Technical Digest, paper JTh5C.9, 2017.

[22] J. M. Ramirez et al., "III-V-on-Silicon Integration: From Hybrid Devices to Heterogeneous Photonic Integrated Circuits," in IEEE Journal of Selected Topics in Quantum Electronics, vol. 26, no. 2, pp. 1-13, MarchApril 2020, Art no. 6100213..

[23] M. A. Tran, D. Huang, J. Guo, T. Komljenovic, P. A. Morton and J. E. Bowers, "Ring-Resonator Based Widely-Tunable Narrow-Linewidth $\mathrm{Si} /$ InP Integrated Lasers," in IEEE Journal of Selected Topics in Quantum Electronics, vol. 26, no. 2, pp. 1-14, March-April 2020, Art no. 1500514.

[24] Hang Guan, Ari Novack, Tal Galfsky, Yangjin Ma, Saeed Fathololoumi, Alexandre Horth, Tam N. Huynh, Jose Roman, Ruizhi Shi, Michael Caverley, Yang Liu, Thomas Baehr-Jones, Keren Bergman, and Michael Hochberg, "Widely-tunable, narrow-linewidth III-V/silicon hybrid external-cavity laser for coherent communication," Opt. Express, 26, 7920-7933, 2018.

[25] R. Pajkovic et al., "Tuning of a widely tunable monolithically integrated InP laser for optical coherence tomography", Proceedings of SPIE, 2019.

[26] L. M. Augustin et al., "InP-Based Generic Foundry Platform for Photonic Integrated Circuits," J. Sel. Top. Quant. Elect., vol. 24, no. 1, 2017.

[27] S. Latkowski et al., "Novel Widely Tunable Monolithically Integrated Laser Source," IEEE Photonics Journal, vol. 7, no. 6, pp. 1-9, Dec. 2015.

[28] S. Andreou, K. A. Williams and E. A. J. M. Bente, "Steady-State Analysis of the Effects of Residual Amplitude Modulation of InP-Based Integrated Phase Modulators in Pound-Drever-Hall Frequency Stabilization," IEEE Photonics Journal, vol. 11, no. 3, pp. 1-14, June 2019.

[29] G. Griffel, "Vernier effect in asymmetrical ring resonator arrays," IEEE Photon. Technol. Lett., 12, 1642-1644, 2000.

[30] http://www.jeppix.eu

[31] F. Rihle, Frequency Standards: Basics and Applications, $1^{\text {st }}$ Edition, Wiley, 2005.

[32] K. Vahala and A. Yariv, "Detuned loading in coupled cavity semiconductor lasers-effect on quantum noise and dynamics," Appl. Phys. Lett., vol. 45, no. 5, 1984.

[33] S. Ogita, M. Yano, H. Ishikawa and H. Imai, "Linewidth reduction in DFB laser by detuning effect," Electronics Letters, vol. 23, no. 8, pp. 393-394, 9 April 1987.

[34] Gianni Di Domenico, Stéphane Schilt, and Pierre Thomann, "Simple approach to the relation between laser frequency noise and laser line shape," Appl. Opt. vol. 49, no. 25, 4801-4807, 2010. 